\title{
Isolement et étude moléculaire de souches des virus de la clavelée et de l'ecthyma contagieux en Tunisie
}

\author{
E. Fakhfakh ${ }^{1}$ C. Le Goff ${ }^{2}$ E. Albina ${ }^{2}$ \\ S. Zekri ${ }^{3}$ C. Seghaier ${ }^{1}$ C. Odisseev ${ }^{1}$ \\ M.H. Jaafoura ${ }^{3}$ S. Hammami ${ }^{1 *}$
}

Mots-clés

Caprin - Ovin - Capripoxvirus - Virus ecthyma contagieux - Parapoxvirus PCR - Phylogénie - Séquence nucléotidique - Tunisie.

\begin{abstract}
Résumé
L'élevage des petits ruminants est touché par plusieurs pathologies infectieuses cutanées. Ainsi, la clavelée et l'ecthyma contagieux représentent deux maladies virales importantes à étudier de part leur allure enzootique et la perte économique qu'elles entraînent. L'objectif de ce travail a été l'isolement en Tunisie de souches virales responsables de ces deux pathologies cutanées et leur caractérisation par l'application et la comparaison de méthodes de diagnostic. La microscopie électronique a été utilisée pour une étude morphologique externe et interne des différentes souches isolées sur culture cellulaire. L'identification par PCR a concerné le gène de la thymidine kinase (TK), le gène de l'analogue du récepteur des chimiokines (CXCR-2) et le gène de la protéine $\mathrm{P} 42 \mathrm{~K}$ présente chez les Parapoxvirus. L'identification moléculaire très sensible et très spécifique des souches de Capripoxvirus a été complétée par une analyse phylogénétique.
\end{abstract}

\section{INTRODUCTION}

L'économie de la plupart des pays du Sud est basée sur l'agriculture et l'élevage. En Afrique du Nord, l'intérêt médical et sanitaire a souvent été porté sur les bovins au détriment des petits ruminants, probablement à cause de leur grande capacité d'adaptation aux conditions difficiles de sécheresse et de pénurie.

En Tunisie, le cheptel ovin compte en moyenne sept millions de têtes. Il est touché par différentes maladies qui constituent une menace pour le développement du secteur agricole. La clavelée et l'ecthyma contagieux sont parmi les affections les plus importantes fréquemment observées en élevage de petits ruminants ; elles revêtent généralement une allure enzootique. L'atteinte par ces deux infections virales cutanées se traduit par une mortalité chez les jeunes animaux, une chute de la reproduction et de la production chez les adultes ; les animaux guéris deviennent généralement des non-valeurs économiques. Devant une telle situation, il est impératif d'instaurer des moyens de diagnostic fiables et rapides

\footnotetext{
1. Institut de la recherche vétérinaire de Tunisie, 14 rue Djebel Lakhdhar, 1006 Tunis, Tunisie

2. Cirad, département Emvt, TA30/G, campus international de Baillarguet, 34398 Montpellier Cedex 5, France

3. Faculté de Médecine de Tunis, rue Djebel Lakhdhar, 1006 Tunis, Tunisie

* Tél. : +21671562602 / 564321 ; fax : +21671569692

E-mail : hammami.salah@iresa.agrinet.tn
}

vis-à-vis de ces deux poxviroses. Cet objectif repose essentiellement sur l'isolement, la caractérisation et l'identification des différentes souches virales responsables ; il constitue également une approche préliminaire pour une étude épidémiologique moléculaire des souches locales de Capripoxvirus.

\section{MATERIEL ET METHODES}

Suite à une investigation réalisée sur l'ensemble du pays, 63 prélèvements ont été obtenus à partir d'ovins présentant des lésions cutanées typiques de clavelée et/ou d'ecthyma contagieux avec parfois atteinte de l'état général. La race, l'âge et le sexe n'ont pas été pris en considération. Les échantillons prélevés étaient composés de papules, de pustules et de croûtes. Ont été comptées respectivement 23 et 40 lésions cutanées douteuses de clavelée et d'ecthyma contagieux. Chaque échantillon a été traité sous forme de filtrat infectieux.

Les techniques expérimentales conduites pour l'identification des isolats suspects de clavelée et d'ecthyma contagieux ont été les mêmes ; elles ont été effectuées en deux étapes décrites ci-après.

\section{Isolement et observation de souches virales}

\section{Culture cellulaire}

Une étape préliminaire de premier et deuxième passage sur culture cellulaire sensible a concerné la totalité des 63 prélèvements. 
Ces premiers essais d'isolement ont été réalisés sur culture primaire de testicules d'agneau (TAP) établie en tapis de cellules âgées de 4 à 5 jours et confluentes à 90 p. 100 . Pour chaque échantillon, une dose d' $1 \mathrm{ml}$ de filtrat infectieux a été inoculée par boîte de culture cellulaire de $25 \mathrm{~cm}^{2}$ préalablement vidée de son milieu d'entretien. Le filtrat d'inoculation a été obtenu à partir du broyage des lésions cutanées à l'aide d'un mortier, et en présence de sable stérile et de milieu (MEM Dubelco, 10 p. 100 SVF), puis filtration de la solution obtenue à $0,45 \mu \mathrm{m}$. Le contact cellules-inoculum a été appliqué durant $1 \mathrm{~h}$ à température ambiante suivi d' $1 \mathrm{~h}$ à $37^{\circ} \mathrm{C}$ dans une étuve à 5 p. 100 de $\mathrm{CO}_{2}$. L'inoculum a ensuite été éliminé, puis le même milieu a été ajouté. La culture a ainsi été maintenue à $37^{\circ} \mathrm{C}$ jusqu'à l'apparition d'un effet cytopathogène (ECP) marqué ou à défaut jusqu'à 12 jours postinoculation. Les échantillons présentant un ECP marquant ont par la suite été inoculés sur des cellules de lignées testiculaires (OA3.Ts) ; le protocole appliqué a été celui décrit précédemment.

\section{Microscopie électronique}

La microscopie électronique a concerné l'examen direct des lésions cutanées et la réalisation de coupes ultra-fines de culture cellulaire inoculée.

L'examen direct a été effectué par imprégnation négative des croûtes et des papules. Chaque lésion a été broyée en présence d'eau bidistillée stérile ; une goutte de cette solution a été déposée sur une grille de microscopie électronique de 200 mesh sans Formvar.
Après un temps de contact de 10 min et séchage, la face traitée de la grille a été contrastée à l'acide phosphotungstique à 2 p. 100, puis observée au microscope.

L'examen sur culture cellulaire inoculée a été réalisé à partir de boite T25 présentant un tapis cellulaire confluant de 95 p. 100 et un ECP prononcé selon le cas. La culture a été rincée et récupérée par grattage en présence de $\mathrm{CaCl} 21 \mathrm{M}$. Le culot cellulaire obtenu après centrifugation a été fixé au glutaraldéhyde à 3 p. 100. Il a ensuite été rincé et immergé dans du tétroxyde d'osmium à 2 p. 100. L'échantillon ainsi déshydraté a été inclus dans un mélange préparé essentiellement à partir d'agar. Cette étape d'inclusion a duré $36 \mathrm{~h}$ et a été suivie d'une polymérisation à l'étuve. L'examen optique a été réalisé sur des coupes ultra-fines de 60 à $70 \mathrm{~nm}$ déposées sur des grilles de 150 mesh. Pour l'observation, un double contraste a été mis en œuvre à base d'acétate d'uranyle à 2 p. 100 et de citrate de plomb à 0,2 p. 100 .

\section{Identification de souches virales}

\section{Réaction de polymérisation en chaîne (PCR)}

L'étape de caractérisation moléculaire a concerné 10 prélèvements de terrain (tableaux I et II) et la souche vaccinale Romania 65 utilisée pour l'immunisation du cheptel ovin national contre la clavelée. La souche de référence Kenya Sheeppox I (KSI), a été choisie comme témoin positif.

\section{Tableau I}

Isolement et identification de souches virales d'ecthyma contagieux

\begin{tabular}{|c|c|c|c|c|c|c|c|}
\hline \multirow[t]{2}{*}{ Inoc } & \multicolumn{2}{|c|}{$\begin{array}{c}\text { Cult. cell. } \\
\text { OA3.Ts }\end{array}$} & \multicolumn{2}{|c|}{$\begin{array}{l}\text { Microsc. } \\
\text { électron. }\end{array}$} & \multicolumn{3}{|c|}{ PCR } \\
\hline & Ecp1 & Ecp2 & Ex. dir. & Ex. ср. & TK & IL8 & P42K \\
\hline E3 & + & ++ & + & + & - & - & + \\
\hline E14 & + & $?$ & + & + & - & - & + \\
\hline E20 & + & + & + & + & - & - & + \\
\hline E31 & + & + & + & + & - & - & + \\
\hline E38 & + & $?$ & + & + & - & - & + \\
\hline
\end{tabular}

Inoc : numéro d'inoculum

Cult. cell. : culture cellulaire

OA3.Ts : lignée cellulaire de testicules d'agneau

Ecp1: effet cytopathogène observé au $1^{\mathrm{er}}$ passage

Ecp 2 : effet cytopathogène observé au $2^{\mathrm{e}}$ passage

++ : effet cytopathogène accentué

+ : effet cytopathogène modéré

? : effet cytopathogène douteux

Microsc. électron. : microscopie électronique

Ex. dir. : examen direct

+ : visualisation de l'aspect enchevêtré caractéristique du virus de l'ecthyma contagieux

Ex. cp. : examen sur coupes de culture cellulaire inoculée

+ : visualisation des différentes étapes de la morphogenèse virale

$\mathrm{PCR}$ : réaction de polymérisation en chaîne

TK : gène codant la thymidine kinase

IL8 : gène codant le récepteur de l'interleukine 8

$\mathrm{P} 42 \mathrm{~K}$ : gène codant la protéine de l'enveloppe des Parapoxvirus

+ : amplification et révélation par électrophorèse horizontale du gène

- : absence d'amplification et de révélation du gène

\section{Tableau II}

Isolement et identification de souches virales de clavelée

\begin{tabular}{lccccccc} 
Inoc & $\begin{array}{c}\text { Cult. cell. } \\
\text { OA3.Ts }\end{array}$ & \multicolumn{3}{c}{$\begin{array}{c}\text { Microsc. } \\
\text { électron. }\end{array}$} & & & PCR \\
& Ecp1 & Ecp2 & Ex. dir. Ex. cp. & TK & IL8 & P42K \\
\hline C4 & ++ & ++ & + & + & + & + & - \\
C9 & ++ & + & + & + & + & + & - \\
C12 & + & $?$ & - & + & + & + & + \\
C13 & $?$ & ++ & + & + & + & + & - \\
C19 & $?$ & $?$ & - & + & + & + & +
\end{tabular}

Inoc : numéro d'inoculum

Cult. cell. : culture cellulaire

OA3.Ts : lignée cellulaire de testicules d'agneau

Ecp1: effet cytopathogène observé au $1^{\text {er }}$ passage

Ecp2 : effet cytopathogène observé au $2^{\mathrm{e}}$ passage

++ : effet cytopathogène accentué

+ : effet cytopathogène modéré

$?$ : effet cytopathogène douteux

Microsc. électron. : microscopie électronique

Ex. dir. : examen direct

+ : visualisation de structures virales de Sheeppox virus

- : absence de visualisation de structures virales de Sheeppox virus

Ex. cp. : examen sur coupe de culture cellulaire inoculée

+ : visualisation des différentes étapes de la morphogenèse virale

PCR : réaction de polymérisation en chaîne

TK : gène codant la thymidine kinase

IL8 : gène codant le récepteur de l'interleukine 8

$\mathrm{P} 42 \mathrm{~K}$ : gène codant la protéine de l'enveloppe des Parapoxvirus

+ : amplification et révélation par électrophorèse horizontale du gène

- : absence d'amplification du gène 
Les gènes ciblés au cours de cette étude ont été :

1. Le gène TK codant la thymidine kinase $(5)$; pour cela, deux couples d'amorces ont été désignés :

- le couple (TK7, TK8) encadrant une région de $680 \mathrm{pb}$

TK7 5'-acttatcagattttgttacgacattg-3'

TK8 5'-cgatgagttctatttccttttctttag-3'

- le couple (TK11, TK12) encadrant une région de $420 \mathrm{pb}$

TK11 5'-ataggacctatgttttctggcaa-3'

TK12 5'-ttagaaaatgcggcgtctttataaca-3'

2. Le gène codant le récepteur analogue à l'interleukine 8 (3); son identification a concerné la portion 3L de longueur 1200 pb amplifiée par cinq amorces déterminées comme suit :

chim1 5'-agtgtatgattaagtaaagcataac-3'

chim2 5'-ttttttattttttatccaatgctaatactacc-3'

chim6 5'-gatgagtattgataggtacctagctgtagt-3'

chim7 5'-cctaaactccgcggtttagcgatactatcg-3'

chim8 5'-atggagtttaggagattgtttgtgt-3'

La localisation de ces amorces sur la portion $3 \mathrm{~L}$ du gène est représentée dans le schéma suivant :

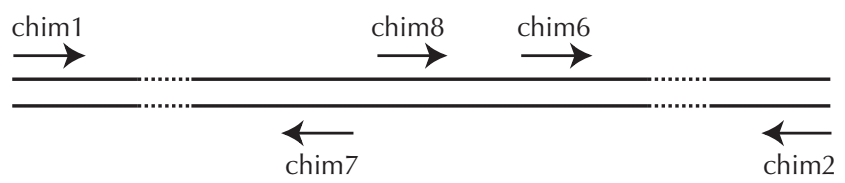

3. Le gène codant B2L de la protéine de l'enveloppe du virus de l'ecthyma contagieux ; la détection a été réalisée par le biais d'un seul couple d'amorces qui encadrait une région de 594 pb. Il a été désigné comme suit (6) :

PPP1 5'-gtcgtccacgatgagcagct-3'

PPP4 5'-tacgtgggaagcgectcgct-3'

La PCR a été réalisée à partir du mélange réactionnel suivant : tampon 10 × $5 \mu \mathrm{l}$, dNTP 100 × 0,5 $\mu \mathrm{l}$, chaque amorce a été utilisée à une concentration de $200 \mathrm{ng} / \mu \mathrm{l}$ à raison de 2 x $1 \mu \mathrm{l}$ par couple d'amorces, la Taq DNA polymérase a été diluée de moitié pour obtenir 2,5 U/Ml. L'ADN à analyser a été extrait avec le kit Qiagen Dneasy Tissue ; il a ensuite été utilisé à une concentration de 50 à $100 \mathrm{ng} / \mu \mathrm{l}$, l'ensemble réactionnel a été ajusté à $50 \mu \mathrm{l}$ avec de l'eau distillée stérile.

La réaction a été effectuée à l'aide d'un appareil Gene Amp PCR system 2400 ; le programme établi a été le même pour les trois détections géniques et n'a différé qu'au niveau de la température d'hybridation des amorces. L'ADN amplifié a été soumis à une électrophorèse horizontale sur gel d'agarose à 1 p. 100 (NuSieve GTG agarose, BMA, Rockland, USA) pour la révélation. Les échantillons présentant une faible concentration d'ADN ont de nouveau été amplifiés par une deuxième PCR. La purification de l'ADN amplifié a été réalisée par électrophorèse horizontale en bande unique sur gel d'agarose ; l'ADN purifié a ensuite été récupéré à l'aide du kit Sephaglass (Amersham Pharmacia).

\section{Etude phylogénétique primaire}

Dans cette partie n'ont été considérés que les isolats de terrain de Capripoxvirus identifiés par PCR et la souche vaccinale Romania 65 (1) ; la liste exhaustive est présentée au tableau III. Le séquençage a concerné la partie amplifiée par (chim2-chim6) de la portion $3 \mathrm{~L}$ du gène codant le récepteur analogue à l'interleukine 8 (IL8). Le produit d'analyse a été séquencé au moins deux fois pour chaque échantillon et comparé à la séquence homologue de la souche de référence KSI (cour 2-6) qui a été elle aussi séquencée.

L'ADN pur a été utilisé à une concentration de 50 ng par échantillon et par réaction. Cette méthode, basée sur celle de Sanger,

\section{Tableau III}

Liste des souches virales utilisées pour les alignements multiples

\begin{tabular}{|c|c|c|}
\hline $\begin{array}{l}\text { Echantillon } \\
\text { séquencé }\end{array}$ & Identifiant & Nature de l'échantillon \\
\hline $\mathrm{C} 3$ & С 3 Р3В & Souche de terrain \\
\hline $\mathrm{C} 4$ & CC4P2B & Souche de terrain \\
\hline C9 & СС9Р2B & Souche de terrain \\
\hline C13 & CC13Р2B & Souche de terrain \\
\hline $\mathrm{C} 17$ & $\mathrm{C} 17 \mathrm{P} 2 \mathrm{~B}$ & Souche de terrain \\
\hline C18 & C18P1B & Souche de terrain \\
\hline $\mathrm{C} 23$ & $\mathrm{C} 23 \mathrm{~B} 2 \mathrm{~B}$ & Souche de terrain \\
\hline C Maroc & CMAROCB & La souche vaccinale Romania 65 \\
\hline Cour 2-6 & COUR2-6B & $\begin{array}{l}\text { La souche de référence Kenya } \\
\text { Sheeppox I (KSI) }\end{array}$ \\
\hline LSDRIL8 & AF325528 & $\begin{array}{l}\text { Séquence homologue du virus de } \\
\text { la dermatose nodulaire contagieuse } \\
\text { (lumpy skin disease), isolat } \\
\text { Neethling } 2490 \text { Kenya }\end{array}$ \\
\hline SPV-K2R & L21931 & $\begin{array}{l}\text { Séquence homologue de la souche } \\
\text { de Swinepox virus Kasza }\end{array}$ \\
\hline SPVRIL8 & AF410153 & $\begin{array}{l}\text { Séquence homologue du Swinepox } \\
\text { virus isolat 17077-99 (USA 1999) }\end{array}$ \\
\hline
\end{tabular}

a été non radioactive et a eu pour principe de base l'utilisation des quatre didésoxynucléotides marqués (kit Big Dye, Perkin Elmer). Le milieu réactionnel a été le suivant :
$\mathrm{x} \mu \mathrm{l}$ d'ADN $50 \mathrm{ng}$
$1 \mu \mathrm{l}$ d'amorce (chim2 ou chim6) à 3,2 pmol/ $\mu \mathrm{l}$
$4 \mu \mathrm{lde}$ mix BDT
$2 \mu \mathrm{l}$ de tampon $5 \mathrm{X}$
$\mathrm{x} \mu \mathrm{l}$ d'eau q.s.p. $20 \mu \mathrm{l}$ de volume total

Le séquençage a été réalisé sur gel de polyacrylamide préchauffé, les échantillons ont été précipités et dénaturés $2 \min 30 \mathrm{~s}$ à $95^{\circ} \mathrm{C}$, puis remis directement dans la glace. La migration a duré $10 \mathrm{~h}$ et s'est faite pendant la nuit ; l'analyse des séquences a été effectuée le lendemain.

L'alignement multiple des séquences en acides nucléiques et en acides aminés a été réalisé à l'aide du logiciel Vector NTI (Invitrogen). Pour une analyse comparative, la séquence homologue de trois poxvirus disponibles a été ajoutée dans les banques de séquences : le virus de la dermatose nodulaire contagieuse ou lumpy skin disease (LSDVRIL8) (13), le Swinepox virus (SPVRIL8) (10) et la souche Kasza (SPV-K2R) (2). Dans les séquences qui suivent, n'ont été notées que les additions ou substitutions de bases par rapport à la séquence consensus (figure 1). La constitution de l'arbre de phylogénie, obtenu sur la base de 1000 répétitions avec le logiciel Phylip, a complété ce travail (figure 2).

\section{RESULTATS}

\section{Ecthyma contagieux}

\section{Culture cellulaire}

Un ECP sur cellules TAP a été observé dans 68 p. 100 des cas au premier passage (17/40) ou au second passage (10/40). Cinq échantillons ont montré des ECP accentués au premier et second passages, ainsi qu'un aspect cellulaire fusiforme caractéristique 


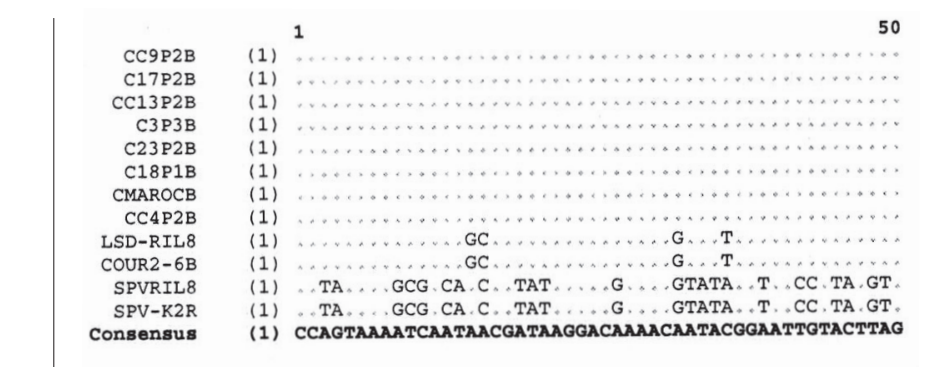

201

\begin{tabular}{ll} 
CC9P2B & $(189)$ \\
C17P2B & $(189)$ \\
\hline$C D P 2 B$ & $(189)$
\end{tabular}

\begin{tabular}{rr} 
C17P2B & $(189)$ \\
CC13P2B & $(189)$ \\
\hline
\end{tabular}

\begin{tabular}{rr} 
CC13P2B & $(189)$ \\
C 3 P $3 \mathrm{~B}$ & $(189)$ \\
\hline
\end{tabular}

С23Р2B $(189)$

C18P1B $(189)$

CMAROCB $(189)$

CC4P2B $(189)$

$\begin{array}{ll}\text { LSD-RIL8 } & (189) \\ \text { COUR2-6B } & (189) \\ \text { S } & \text { (18) }\end{array}$

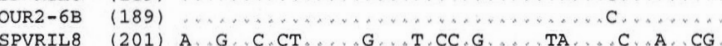

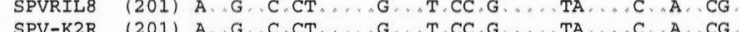

Consensus (201) TGAAATAAACATATTTGGAATGATTATACCGCTAATTATTTGGCTATATT

250
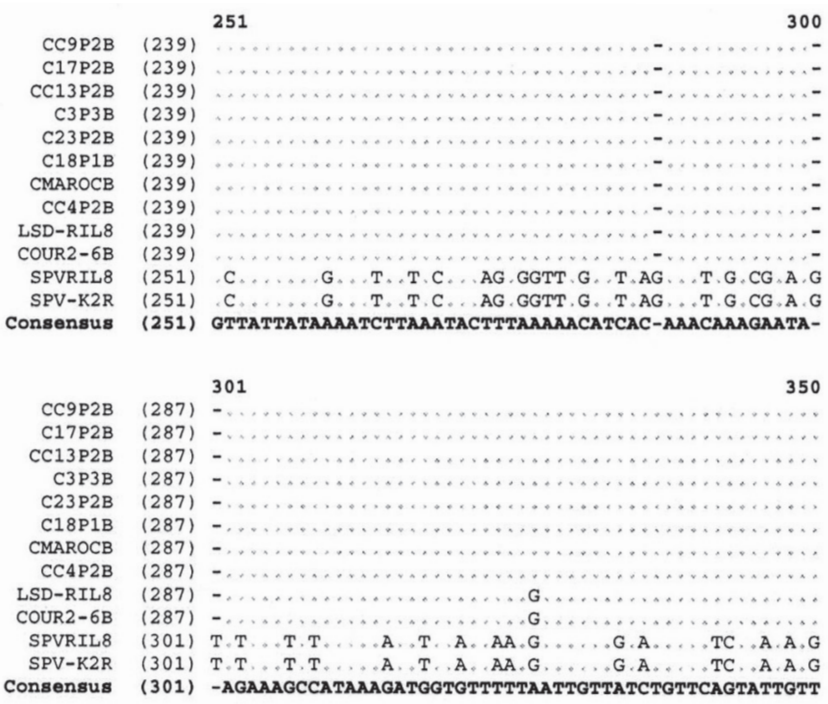

351

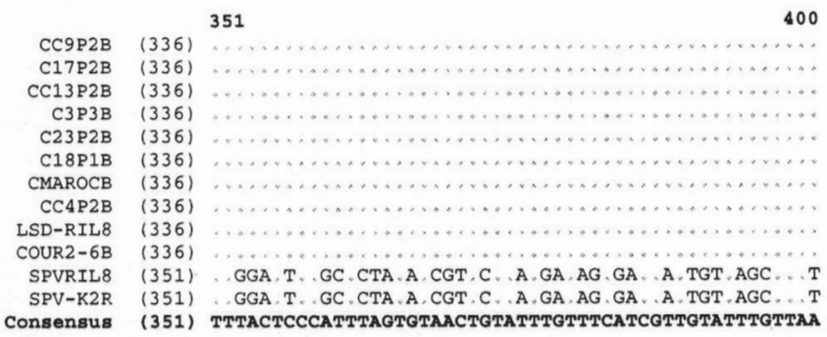

401

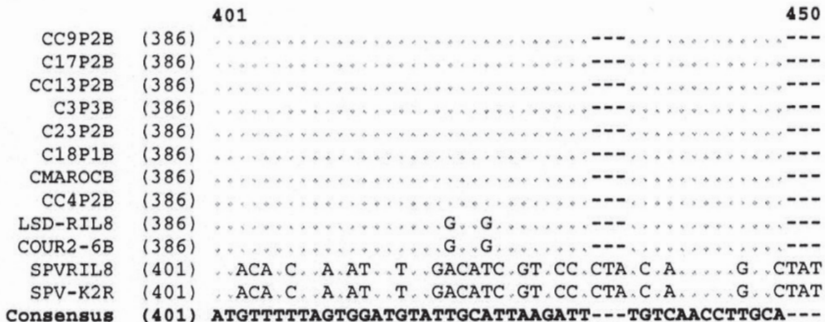

451

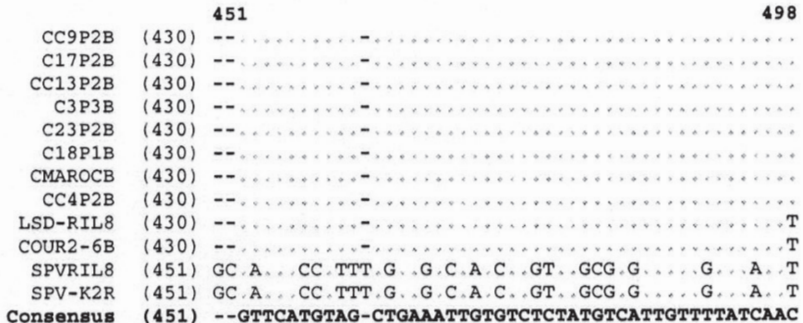

Figure 1 : alignement multiple des séquences en acides nucléiques du virus de la clavelée.

d'une inoculation par le virus de l'ecthyma contagieux (11). Ces cinq prélèvements ont fait l'objet d'une deuxième inoculation sur des cellules lignées de testicules d'agneaux OA3.Ts. Le résultat obtenu a différé par la présence d'ECP douteux (tableau I).

\section{Microscopie électronique}

Cette technique a été appliquée sur cinq prélèvements ayant produit un ECP (tableau I). Le contraste négatif à l'acide phosphotungstique a révélé la présence du virus de l'ecthyma contagieux dans la totalité des prélèvements (figure 3). L'observation de coupes ultra-fines a montré les différentes étapes de la morphogenèse virale (figure 4).

\section{PCR}

Cette technique n'a montré une amplification positive que pour le gène codant de la protéine de l'enveloppe $\mathrm{P} 42 \mathrm{~K}$ (tableau I) (figure 5).

\section{Clavelée}

\section{Culture cellulaire}

Parmi les seize échantillons (70 p. 100 de positifs) avec ECP au premier et/ou second passage, cinq échantillons ont été sélectionnés pour adaptation sur cellules OA3.Ts. Parmi ces cinq, trois isolats seulement ont développé un ECP caractéristique des poxvirus (tableau II). Les cellules infectées avaient une forme arrondie avec un aspect interne granuleux, témoin de la présence de particules virales dans le cytoplasme cellulaire (9).

\section{Microscopie électronique}

L'examen direct des papules a permis de visualiser trois échantillons positifs (tableau II) (figure 6). L'échantillon C12 a présenté une structure enchevêtrée en forme de cocon caractéristique 


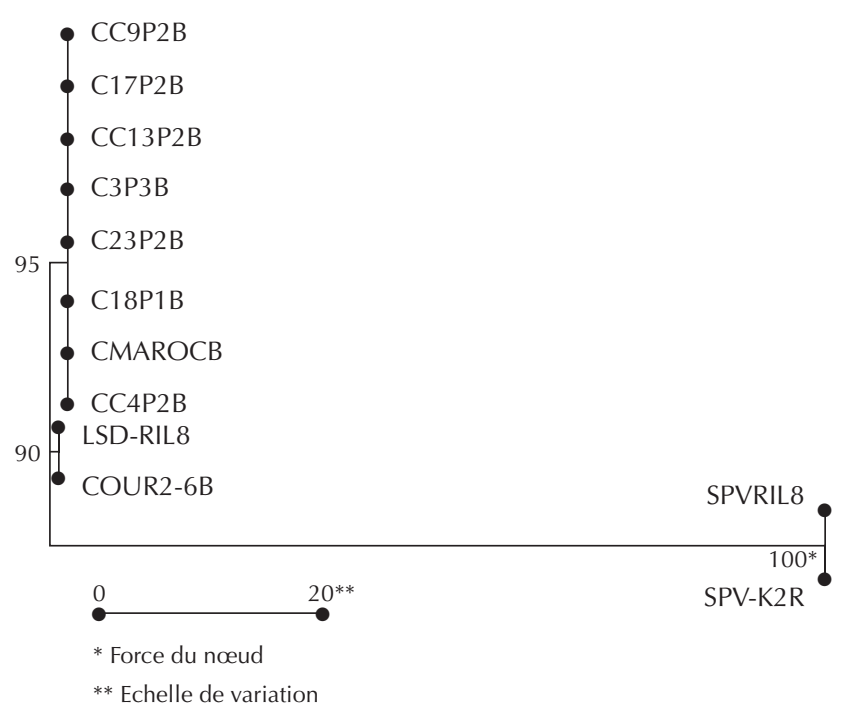

Figure 2: arbre phylogénétique des séquences. L'échelle indique le pourcentage de substitutions en nucléotides.
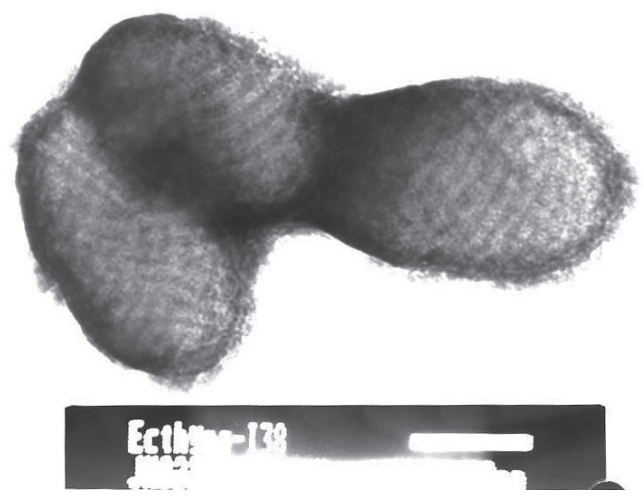

Figure 3 : particule virale d'ecthyma contagieux observée en coloration négative par microscopie électronique (laboratoire d'Embryo-histo-anatomopathologie, faculté de Médecine de Tunis) ; x 40K; barre : $200 \mathrm{~nm}$. A noter la forme de cocon et l'aspect enchevêtré typique au sein des poxvirus.

des Parapoxvirus et aucune structure virale n'a pu être identifiée au niveau de l'échantillon C19. L'étude des coupes ultra-fines a mis en évidence les différentes étapes de morphogenèse virale pour les cinq prélèvements suspects (figure 7).

\section{PCR}

Les résultats de l'amplification génique ont été visualisés pour le gène de la thymidine kinase et le gène du récepteur analogue à l'IL8 (tableau II, figure 8). Néanmoins, les échantillons C12 et C19 n'ont présenté une amplification positive que pour le gène $\mathrm{P} 42 \mathrm{~K}$.

\section{Séquençage}

La longueur d'ADN séquencée obtenue n'a pas été la même pour tous les échantillons (tableau IV, figure 1). Le séquençage a permis de mettre en évidence trois groupes distincts de séquences. Le premier était constitué par les isolats de terrain et la souche vaccinale Romania. Ce groupe a présenté un taux de substitution en nucléotides et en acides aminés inférieur à 5 p. 100. Le second groupe était représenté par la séquence homologue (cour 2-6) de la souche KS1 et celle de la souche LSDVRIL8. En revanche, des taux de substitution en nucléotides et en acides aminés de 33,2 et 65,5 p. 100 ont été observés avec les deux souches des Swinepox virus constituant le troisième groupe (figure 2).

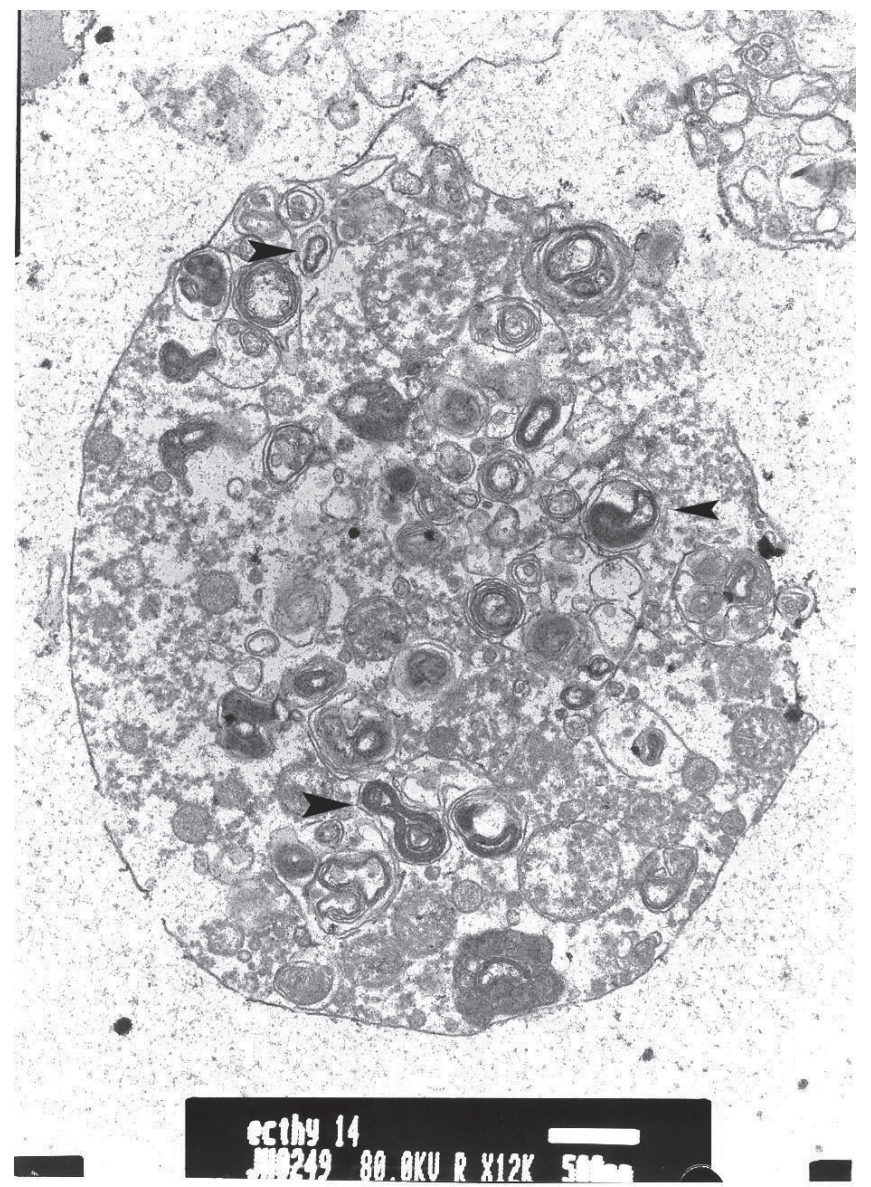

Figure 4 : particule virale d'ecthyma contagieux observée en coloration négative par microscopie électronique (laboratoire d'Embryo-histo-anatomopathologie, faculté de Médecine de Tunis) ; x 12K ; barre : $500 \mathrm{~nm}$. Les flèches indiquent les différentes étapes de morphogenèse virale observées sur coupes ultra-fines cellulaires inoculées par le virus de l'ecthyma contagieux.

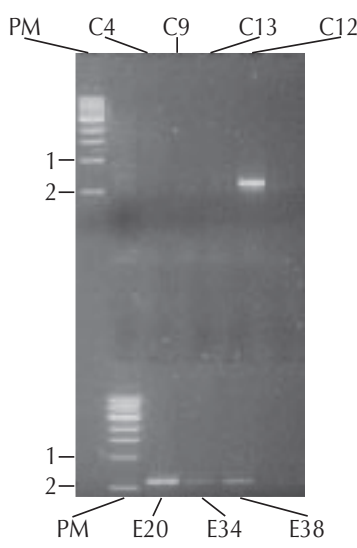

Figure 5: amplification d'une portion de 594 pb du gène de la protéine $\mathrm{P} 42 \mathrm{~K}$ de l'enveloppe du virus de l'ecthyma contagieux.

PM : poids moléculaire. E20, E34, E38: isolats suspects d'ecthyma contagieux confirmés positifs par microscopie électronique. C4, C9, C13 : isolats suspects de clavelée confirmés positifs par microscopie électronique. C12 : isolat suspect de clavelée et confirmé négatif par microscopie électronique. Electrophorèse horizontale sur gel d'agarose à $1 \%$ TAE. $1=1000 \mathrm{pb} ; 2=500 \mathrm{pb}$. 


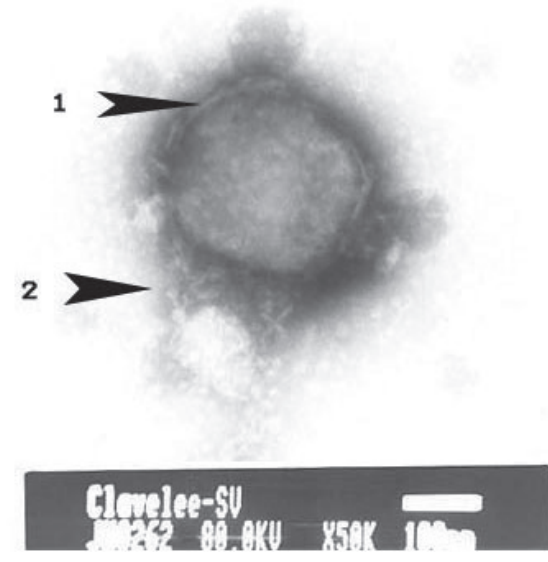

Figure 6 : particule virale de Sheeppox observée en coloration négative par microscopie électronique (laboratoire d'Embryo-histo-anatomopathologie, faculté de Médecine de Tunis) ; x 50K; barre : $100 \mathrm{~nm} .1$ : capsule virale. 2 : virus du sheep pox; forme rectangulaire à angles arrondis.

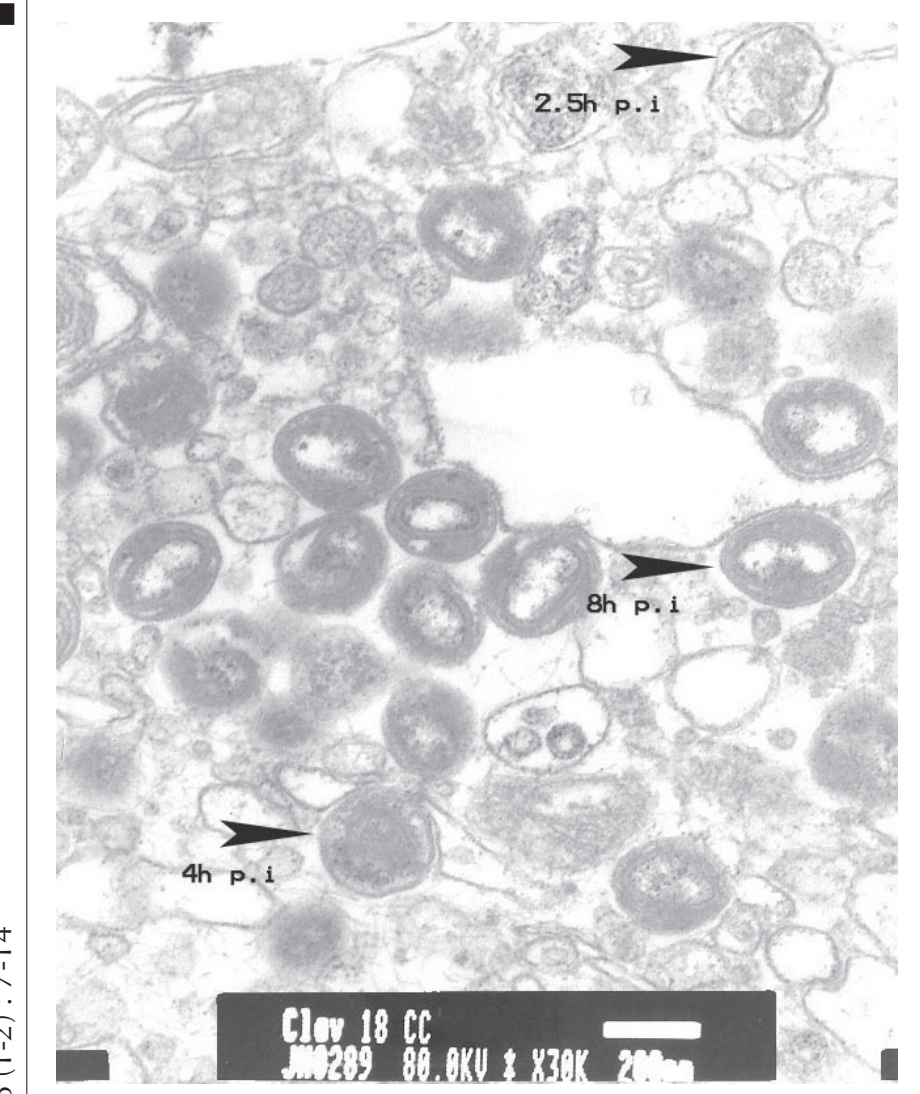

Figure 7 : coloration positive de particules virales de Sheeppox virus en cours de morphogenèse (laboratoire d'Embryo-histo-anatomopathologie, faculté de Médecine de Tunis). p.i : postinoculation ; x 30K; barre : $200 \mathrm{~nm}$.

\section{- DISCUSSION}

L'enquête épidémiologique nationale menée par l'Institut de la recherche vétérinaire de Tunis en collaboration avec les services du Commissariat régional de développement agricole a représenté la partie appliquée. Son but était, d'une part, d'isoler des souches de Capripoxvirus et de Parapoxvirus, et de les identifier par différents moyens de diagnostic, et, d'autre part, de contribuer à

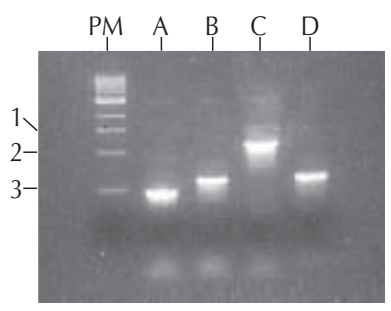

Figure 8: amplification spécifique $d u$ gène de la TK par le couple d'amorces D. Amplification spécifique du gène du récepteur analogue à I'IL8 par le biais des trois couples d'amorces $A, B, C$. Exemple : isolat $n^{\circ} 9$ suspect clavelée. $P M$ : poids moléculaire. A : amplification par (chim1, chim7) de longueur 495 pb. B : amplification par (chim2, chim6) de longueur 650 pb. C: amplification par (chim1, chim2) de longueur 1200 pb. D : amplification par (TK7, TK8) de longueur 680 pb. Electrophorèse horizontale sur gel d'agarose à $1 \%$ TAE. $1=1500 \mathrm{pb} ; 2=1000 \mathrm{pb} ; 3=500 \mathrm{pb}$.

\section{Tableau IV}

Longueur des fragments obtenus après séquençage

\begin{tabular}{lccc} 
Echantillon & $\begin{array}{c}\text { Nb. } \\
\text { séquençages }\end{array}$ & chim2 $(\mathbf{p b})$ & chim6 (pb) \\
\hline C3 & 3 & 580 & 590 \\
C4 & 3 & 570 & 590 \\
C9 & 3 & 590 & 580 \\
C13 & 2 & 590 & 600 \\
C17 & 2 & 590 & 600 \\
C18 & 2 & 550 & 600 \\
C23 & 2 & 577 & 600 \\
C Maroc & 2 & 550 & 560 \\
cour 2-6 & 2 & 560 & 600
\end{tabular}

C3, C4, C9, C13, C17, C18, C23 : isolats de terrain identifiés par PCR

C Maroc : la souche vaccinale Romania 65

cour 2-6 : la souche de référence Kenya Sheeppox I (KSI)

une étude épidémiologique moléculaire sur une partie du génome des souches de Capripoxvirus isolées sur le terrain.

L'isolement viral sur culture cellulaire primaire a concerné un échantillonnage de 63 prélèvements (croûtes, papules) provenant d'animaux suspects de clavelée ou d'ecthyma contagieux. Les deux virus ont présenté les mêmes affinités cellulaires. Ils ont produit un ECP à partir du cinquième jour évoluant jusqu'au douzième jour postinoculation.

Le premier passage sur TAP a révélé la présence d'un ECP dans 43 p. 100 des cas suspects d'ecthyma contagieux et dans 22 p. 100 des cas suspects de clavelée. Au deuxième passage ces valeurs ont atteint respectivement 68 et 70 p. 100 . La nature des résultats obtenus n'a confirmé en aucun cas la présence ou l'absence d'une souche virale suspectée préalablement. Ceci restait conditionné par la faculté d'adaptation de l'agent infectieux à son nouvel environnement cellulaire. Néanmoins, l'action de certains interférons cellulaires peut aussi entraîner la perte d'une souche isolée sur culture cellulaire (11).

A l'issue de cette première étude, 10 échantillons ont été sélectionnés (5 suspects d'ecthyma contagieux et 5 suspects de clavelée) 
pour adaptation sur des cellules lignées de testicules d'agneaux OA3.Ts. Les résultats ont différé par la présence de cas douteux et une faible intensité d'ECP. Selon Ramisse et coll. (12), le système de réplication de choix des poxvirus est représenté par les cellules primaires de testicules et de rein d'agneau et les cellules d'embryon total de mouton.

La microscopie électronique a appuyé les résultats observés en culture cellulaire, notamment pour les dix échantillons choisis. L'examen direct par contraste négatif a confirmé que les ECP observés étaient dus à des isolats suspects d'ecthyma contagieux ou de clavelée. D'autre part, trois échantillons seulement ont présenté des structures virales semblables à celle des Capripoxvirus. On souligne que cette technique ne permet pas la distinction morphologique entre les Capripoxvirus et les autres poxvirus. A défaut, la visualisation de l'aspect enchevêtré caractéristique du virus de l'ecthyma contagieux met à profit cette application optique. L'examen de coupes ultra-fines à partir de culture cellulaire inoculée est plus spécifique. En effet, cette technique a montré un résultat positif sur la totalité des prélèvements, y compris l'échantillon C19 qui a présenté un résultat douteux par contraste négatif.

L'identification génomique par PCR des dix isolats traités a concerné le gène de la thymidine kinase, le gène $\mathrm{P} 42 \mathrm{~K}$ codant la protéine d'enveloppe de l'ecthyma contagieux et le gène de l'analogue au récepteur à l'IL8. La spécificité et la sensibilité de la PCR reposent essentiellement sur le choix des amorces. L'utilisation de cette technique a été largement appuyée par les travaux d'Ireland et Binepal (7), et Inoshima et coll. (6) pour l'identification des Capripoxvirus et des Parapoxvirus.

L'utilisation respective des deux couples d'amorces (TK7, TK8) et (TK11, TK12) n'a révélé une amplification de 680 pb et de 420 pb que pour les échantillons suspects de clavelée. Le gène TK a présenté une topographie conservée au sein de la majorité des poxvirus (5) à l'exception des Parapoxvirus où il est absent (14). D'autre part, l'identification du gène $\mathrm{P} 42 \mathrm{~K}$ a été constatée pour tous les isolats suspects d'ecthyma contagieux mais aussi pour l'échantillon C12 suspect de clavelée.

L'identification du gène analogue du récepteur à l'IL8 a intéressé la principale partie de ce travail. L'IL8 est un membre de la sous-famille des chimiokines de type C-X-C ; il intervient dans la réponse immunitaire en stimulant l'activité des neutrophiles (8). L'identification de ce gène a concerné la portion 3L du fragment HindIII Q2 du génome de la souche KSI (3). La détection a été réalisée par le biais de quatre couples d'amorces. Le couple d'amorces (chim2 chim6) a fonctionné sur la totalité des échantillons. Selon les travaux de Cao et coll. (3), les résultats obtenus ont confirmé l'isolement de souches de Capripoxvirus pour lesquelles la région Q2/3L identifiée ne figure qu'en une seule copie. Par comparaison, le Swinepox virus renfermait dans son génome deux phases de lecture identiques K2R et C3L situées chacune au niveau d'une extrémité terminale inversée ITR. La phase K2R était homologue au récepteur IL8-RB (CXC-R2) (10). Cette phase a servi d'échelle pour les alignements multiples de la partie séquençage. Concernant les isolats suspects en matière d'ecthyma contagieux, aucune amplification n'a été détectée. Ceci pouvait être attribué à la position du gène à l'extrémité terminale du génome. En effet, chez les Parapoxvirus les régions ITR sont très instables. Il y siège un phénomène de transposition-délétion capable d'entraîner la perte de la séquence recherchée (4).

Le fragment (chim2-chim6) identifié sur tous les échantillons a été séquencé. L'analyse phylogénétique de la souche de Swinepox virus SPVRIL8 et celle de LSDVRIL8 ont montré un faible pourcentage de substitutions en acides aminés (2). Toutes les séquences alignées ont été bornées sur la base des travaux de Cao et coll. (3).
La phase K2R homologue au récepteur IL8-RB (CXC-R2) (10) a servi d'échelle pour les alignements multiples de la partie séquençage. Ainsi, les Swinepox virus ont permis d'ancrer l'arbre phylogénique. Sur ce dernier, trois groupes distincts de séquences ont été observés. Un lien de parenté ou de dissociation a été représenté entre les groupes. Les isolats de terrain et la souche Romania 65 ont formé un ensemble homogène et ceci malgré la diversité de leur origine géographique. Ce groupe a présenté un taux de substitutions en nucléotides inférieur à 5 p. 100 avec la séquence homologue de la souche KSI (cour 2-6) et celle de la souche LSDVRIL8. En revanche, une grande divergence nucléotidique de 33,2 p. 100 a été observée avec les deux souches de Swinepox virus. Ceci reste cohérent puisque ces dernières appartiennent à un genre différent (10). La conversion des séquences en acides aminés a montré un taux de variation de 5 p. 100 entre les isolats tunisiens et la souche de référence KSI (cour 2-6). Ceci a résulté de sept substitutions d'acides aminés sur 158 dues à sept mutations de bases. Quatre mutations de bases étaient silencieuses.

Les résultats obtenus ont montré la conservation de cette séquence au sein du genre des Capripoxvirus, et ceci malgré la diversité topographique des échantillons. Cette première approche incite à approfondir cette analyse sur un fragment plus long du gène appartenant à des isolats de diverses origines provenant de différentes espèces animales. Un échantillonnage ainsi constitué permettra de vérifier si cette portion de gène pourrait être un traceur épidémiologique des souches de Capripoxvirus.

\section{CONCLUSION}

Ce travail a abouti à l'identification de souches virales sauvages de Capripoxvirus et de Parapoxvirus. Chaque outil de diagnostic a présenté des avantages et des inconvénients vis-à-vis de la nature de l'infection suspectée. Ainsi la microscopie électronique reste la méthode de choix pour la révélation optique d'un Parapoxvirus par rapport à un Capripoxvirus. L'utilisation de la PCR pour la recherche de Capripoxvirus et de Parapoxvirus a été appuyée par plusieurs exemples. La détection du gène de la TK et de celui du récepteur analogue à l'IL8 a été possible sur toutes les souches suspectes appartenant au genre Capripoxvirus. En revanche, le gène codant pour la protéine virale de l'enveloppe de l'ecthyma contagieux (la P42K) a été identifié seulement dans les souches de Parapoxvirus. D'autre part, le profil génétique des souches de terrain de Capripoxvirus concernant la portion du gène analogue du récepteur de l'IL8 a été identique à celui de la souche vaccinale Romania 65, souche d'origine marocaine. Par ailleurs, le profil des souches de terrain a été très proche de celui de la souche vaccinale KSI. Ces résultats mettent en évidence la conservation de cette portion génique malgré la diversité géographique des souches considérées. Ceci incite donc à approfondir l'étude et la comparaison avec d'autres séquences du génome de ces souches.

\section{BIBLIOGRAPHIE}

1. ACHOUR H.A., BOUGUEDOUR R, BOUHBAL A, GUECHTOUL A., AOUISSAT M., 2000. Etude comparative du pouvoir immunisant de quelques souches atténuées de virus de la clavelée et d'un vaccin sensibilisé. Revue sci. tech. Off. int. Epizoot., 19 : 773-783.

2. AFONSO C.L., TULMAN E.R., LU Z., BALINSKY C., OSORIO F.A., ZSAK L., KUTISH G.F., ROCK D.L., 2002. The genome of Swinepox virus. J. Virol., 76: 783-790.

3. CAO J.X., GERSHON P.D., BLACK D.N., 1995. Sequence analysis of HindIII Q2 fragment of Capripoxvirus reveals a putative gene encoding a G-protein-coupled chemokine receptor homologue. Virology, 209: 207-212. 
4. FLEMING S.B., LYTTLE D.J., SULLIVAN J.T., MERCER A.A., ROBINSON A.J., 1995. Genomic analysis of a transposition-deletion variant of orf $v$ reveals a $3.3 \mathrm{kbp}$ region of non-essential DNA. J. gen. Virol., 76: 2969-2978.

5. GERSHON P.D., BLACK D.N., 1989. The nucleotide sequence around the Capripoxvirus thymidine kinase gene reveals a gene shared specifically with Leporipoxvirus. J. gen. Virol., 70: 525-533.

6. INOSHIMA Y., MOROOKA A., SENTSUI H., 2000. Detection and diagnosis of Parapoxvirus by the polymerase chain reaction. J. virol. Methods, 84: 201-207.

7. IRELAND D.C., BINEPAL Y.S., 1998. Improved detection of Capripoxvirus in biopsy samples by PCR. J. virol. Methods, 74: 1-7.

8. LALANI A.S., NESS T.L., SINGH R., HARRISSON J.K., SEET B.T., KELVIN D.J., MCFADDEN G., MOYER R.W., 1998. Functional comparisons among members of the poxvirus T1/35 kDa family of soluble CC-chemokine inhibitor glycoproteins. Virology, 250: 173-184.

9. LEFEVRE P.C., 1983. La variole ovine (clavelée) et la variole caprine. Maisons-Alfort, France, Gerdat-lemvt, $171 \mathrm{p}$.

\section{Summary}

Fakhfakh E., Le Goff C., Albina E., Zekri S., Seghaier C., Odisseev C., Jaafoura M.H., Hammami S. Isolation and Molecular Study of Sheep Pox and Orf Viruses in Tunisia

Small ruminant farms are affected by several infectious cutaneous diseases. Thus, sheep pox and contagious pustular dermatitis represent two major viral diseases that need investigating as they can reach endemic proportions and cause important economic losses. The aim of this work was to isolate the viral strains at the origin of both diseases in Tunisia, and to characterize them by applying and comparing several diagnosis techniques. Electronic microscopy was used to study the external and internal morphology of the various strains isolated on cell culture. Identification by PCR concerned the thymidine kinase (TK) gene, the IL8 receptor-like gene specific of Capripoxvirus, and the P42K gene specific of Parapoxvirus. The very sensitive and very specific molecular identification of Capripoxvirus wild strains was completed by a phylogenetic analysis.

Keywords: Goat - Sheep - Capripoxvirus - Contagious ecthyma virus - Parapoxvirus - PCR - Phylogeny - Nucleotide
10. MASSUNG R.F., JAYARAMA V., MOYER R.W., 1993. DNA sequence analysis of conserved and unique regions of Swinepox virus: identification of genetic elements supporting phenotypic observations including a novel $G$ protein-coupled receptor homologue. Virology, 197: 511-528.

11. PRECAUSTA P., 1974. Clavelée. In : Bricout F., Joubert L., Huraux J.M., éds, Manuel de diagnostic séro-immunologique des viroses humaines et animales, 1 ère édn. Paris, France, Maloine, p. 239-241.

12. RAMISSE J., ASSO J., HASSANI A., ANANE O., JEMLI J., 1978. Culture du virus claveleux sur cellules : application à la vaccination et au contrôle de l'immunité. Revue Elev. Méd. vét. Pays trop., 31 : 11-19.

13. TULMAN E.R., AFONSO C.L., LU Z., ZSAK L., KUTISH G.F., ROCK D.L., 2001. Genome of lumpy skin disease virus. J. Virol., 75: 7122-7130.

14. VAN REGENMORTEL M.H., FAUQUET C.M., BISHOP D.H.L., 2000. Poxviridae. In: virus taxonomy classification and nomenclature of viruses. Seventh report of the international committee on taxonomy of viruses. San Diego, CA, USA, Academic Press, p. 137-157.

Reçu le 01.04.2004, accepté le 15.06.2005

\section{Resumen}

Fakhfakh E., Le Goff C., Albina E., Zekri S., Seghaier C., Odisseev C., Jaafoura M.H., Hammami S. Aislamiento y estudio molecular de cepas de virus de la viruela (lengua azul) y del ectima contagioso en Tunisia

La cría de los pequeños rumiantes está siendo afectada por varias patologías infecciosas cutáneas. Así, la viruela y el ectima contagioso representan dos enfermedades virales importantes a estudiar, a causa de su aspecto enzoótico y por la pérdida económica que conllevan. El objetivo del presente estudio fue el aislamiento en Tunisia de cepas virales responsables de estos dos problemas cutáneos y su caracterización mediante la aplicación y la comparación de métodos de diagnóstico. Se utilizó la microscopía electrónica para un estudio morfológico externo e interno de las diferentes cepas aisladas a partir del cultivo celular. La identificación mediante PCR concernió el gen de la timidin-quinasa (TK), el gen del receptor análogo de las quimokinas (CXCR-2) y el gen de la proteína P42K presente en los Parapoxvirus. La identificación molecular muy sensible y específica de las cepas de Capripoxvirus fue completada mediante un análisis filogenético.

Palabras clave: Caprino - Ovino - Capripoxvirus - Virus ectima contagioso - Parapoxvirus - PCR - Filogenía - Secuencia nucleotídica - Túnez. 